\title{
Erratum
}

\section{Erwin Bünning (1906-1990): A centennial homage}

M K Chandrashekaran 2006, J. Biosci., 31, 5-12

As printed in the March 2006 issue, the first reference in the list that follows the article is wrong. The correct reference is given below.

Bünning E 1975 Wilhelm Pfeffer: Apotheker, Chemiker, Botaniker, Physiologe 1845-1920 (Wissenschaftliche Verlagsgesellschaft) 